\title{
EUCLIDEAN SCISSOR CONGRUENCE GROUPS AND MIXED TATE MOTIVES OVER DUAL NUMBERS
}

\author{
A. B. Goncharov
}

\begin{abstract}
We define Euclidean scissor congruence groups for an arbitrary algebraically closed field $F$ and formulate a conjecture describing them. Using the Euclidean and Non-Euclidean $F$-scissor congruence groups we construct a category which is conjecturally equivalent to a subcategory of the category $\mathcal{M}_{T}\left(F_{\varepsilon}\right)$ of mixed Tate motives over the dual numbers $F_{\varepsilon}:=F[\varepsilon] / \varepsilon^{2}$.
\end{abstract}

To Spencer Bloch, with admiration, for his 60th birthday

\section{Introduction}

\subsection{Euclidean scissor congruence groups and a generalization of}

Hilbert's third problem. Let $F$ be an arbitrary algebraically closed field. In Chapter 3 of [8] we defined an $F$-scissor congruence group $S_{n}(F)$ of polyhedrons in the projective space $P^{2 n-1}(F)$ equipped with a non-degenerate quadric $Q$. The classical spherical and hyperbolic scissor congruence groups are subgroups of $S_{n}(\mathbb{C})$. The direct sum

$$
S_{\bullet}(F):=\oplus_{n \geq 0} S_{n}(F) ; \quad S_{0}(F)=\mathbb{Q}
$$

is equipped with a structure of a commutative, graded Hopf $\mathbb{Q}$-algebra. The coproduct is given by the Dehn invariant map

$$
D: S_{n}(F) \longrightarrow \oplus_{0 \leq k \leq n} S_{k}(F) \otimes S_{n-k}(F)
$$

In this paper we define Euclidean $F$-scissor congruence groups $\mathcal{E}_{n}(F)$ of polyhedrons in $(2 n-1)$-dimensional affine space over $F$ equipped with a nondegenerate quadratic form $Q$. If $F=\mathbb{R}$ (which is not algebraically closed!) and $Q$ is positive definite, we get the classical Euclidean scissor congruence group $\mathcal{E}_{n}(\mathbb{R})$ in $\mathbb{R}^{2 n-1}$. We define the Dehn invariant map

$$
D^{E}: \mathcal{E}_{n}(F) \longrightarrow \oplus_{1 \leq k \leq n} \mathcal{E}_{k}(F) \otimes S_{n-k}(F)
$$

and show that it provides the graded $\mathbb{Q}$-vector space

$$
\mathcal{E}_{\bullet}(F):=\oplus_{k \geq 0} \mathcal{E}_{n}(F)
$$

with a structure of a comodule over the Hopf algebra $S_{\bullet}(F)$. The cobar complex calculating the cohomology of this comodule looks as follows:

$$
\mathcal{E}_{\bullet}(F) \longrightarrow \mathcal{E}_{\bullet}(F) \otimes S_{\bullet}(F) \longrightarrow \mathcal{E}_{\bullet}(F) \otimes S_{\bullet}(F)^{\otimes 2} \longrightarrow \ldots
$$

Received February 19, 2004. 
The differential is cooked up from $D^{E}$ and $D$ using the Leibniz rule. For example the first two arrows are $D^{E}$ and $D^{E} \otimes \mathrm{Id}-\mathrm{Id} \otimes D$.

We place the first group in degree 1 , denote the complex by $\mathcal{E}_{(\bullet)}^{*}(F)$, and call it Euclidean Dehn complex. The differential preserves the grading. We denote by $\mathcal{E}_{(n)}^{*}(F)$ the degree $n$ subcomplex of (1).

The action by dilatations of the group $F^{*}$ in a Euclidean $F$-vector space provides an $F^{*}$-action on the Euclidean $F$-scissor congruence groups. So (1) is a complex of $F^{*}$-modules. For an $F$-vector space $V$ denote by $V\langle p\rangle$ the twisted $F^{*}$-module structure $*$ on $V$ given by $f * v=f^{2 p+1} \cdot v$. The volume provides a homomorphism

$$
\mathrm{Vol}: \mathcal{E}_{n}(F) \longrightarrow F\langle n-1\rangle
$$

Conjecture 1.1. a) Let $F$ be an arbitrary algebraically closed field. Then we have a canonical isomorphism of $F^{*}$-modules

$$
H^{i}\left(\mathcal{E}_{(n)}^{*}(F)\right)=\Omega_{F / \mathbb{Q}}^{i-1}\langle n\rangle
$$

b) The same is true in the classical case $F=\mathbb{R}$.

One can view this as a generalization of Hilbert's Third Problem. Indeed, according to Sydler's theorem [16] the following complex is exact:

$$
0 \longrightarrow \mathbb{R} \longrightarrow \mathcal{E}_{2}(\mathbb{R}) \stackrel{D^{E}}{\longrightarrow} \mathbb{R} \otimes S^{1} \longrightarrow \Omega_{\mathbb{R} / \mathbb{Q}}^{1} \longrightarrow 0
$$

In particular the kernel of the Dehn invariant is identified by the volume homomorphism with $\mathbb{R}$. So the Dehn invariant and the volume determine a polyhedron in $\mathbb{R}^{3}$ uniquely up to scissor congruence.

Sydler's theorem gives the $n=2$ case of the part b) of conjecture. The $n=2$ case of the part a) can probably be deduced from the results of Sydler, Dupont, Cathelineau, Sah in [16], [6], [7], [3].

Another key problem is the structure of the groups $\mathcal{E}_{n}(F)$. By the Euler characteristic argument (and induction) the answer is controlled, although in a cryptic way, by Conjecture 1.1. Let us try to get a conjectural answer in a more explicit form.

\subsection{A hypothetical description of the Euclidean scissor congruence groups. Let}

$$
\mathcal{Q} \bullet(F):=\frac{S_{\bullet}(F)}{S_{>0}(F) \cdot S_{>0}(F)}
$$

be the space of indecomposables of the Hopf algebra $S_{\bullet}(F)$. It is a graded Lie coalgebra with the cobracket induced by the coproduct in $S_{\bullet}(F)$.

Let $\mathbb{Q}_{\varepsilon}-$ mod be the tensor category with the objects $V=V_{0} \oplus V_{1} \varepsilon$, where $V_{0}, V_{1}$ are $\mathbb{Q}$-vector spaces, and Hom and tensor product defined by

$$
\begin{gathered}
\operatorname{Hom}_{\mathbb{Q}_{\varepsilon}-\text { mod }}\left(V_{0} \oplus V_{1} \varepsilon, W_{0} \oplus W_{1} \varepsilon\right):=\operatorname{Hom}\left(V_{0}, W_{0}\right) \oplus \operatorname{Hom}\left(V_{1}, W_{1}\right) \\
\left(V_{0} \oplus V_{1} \varepsilon\right) \otimes_{\mathbb{Q}_{\varepsilon}-m o d}\left(W_{0} \oplus W_{1} \varepsilon\right):=V_{0} \otimes W_{0} \oplus\left(V_{0} \otimes W_{1} \oplus W_{0} \otimes V_{1}\right) \varepsilon
\end{gathered}
$$

Since $V_{1} \varepsilon \otimes V_{2} \varepsilon=0$, it is not a rigid tensor category. 
Observe that a Lie coalgebra $\mathcal{L}_{\varepsilon}$ in the category $\mathbb{Q}_{\varepsilon}-\bmod$ is just the same thing as a Lie coalgebra $\mathcal{L}$ and a comodule $\mathcal{L}^{a}$ over it: $\mathcal{L}_{\varepsilon}=\mathcal{L} \oplus \mathcal{L}^{a} \cdot \varepsilon$.

Recall that the Euclidean $F$-scissor congruence groups are organized into a comodule $\mathcal{E}_{\bullet}(F)$ over $S_{\bullet}(F)$, and hence over $\mathcal{Q}_{\bullet}(F)$. Therefore combining the Euclidean and Non-Euclidean $F$-scissor congruence groups we get a Lie coalgebra

$$
\mathcal{Q}_{\bullet}\left(F_{\varepsilon}\right):=\mathcal{Q}_{\bullet}(F) \oplus \mathcal{E}_{\bullet}(F) \cdot \varepsilon
$$

in the category $\mathbb{Q}_{\varepsilon}$-mod. One has $\mathcal{Q}_{1}(F)=S_{1}(F)=F^{*}$ and $\mathcal{E}_{1}(F)=F$.

Recall the higher Bloch groups $\mathcal{B}_{n}(F)$ ([9]-[10]). We also need their additive versions, the $F^{*}$-modules $\beta_{n}(F)$, defined in Section 3 as extensions of Cathelineau's groups [3]. The $F^{*}$-module $\beta_{2}(F)$ is isomorphic to the one defined by $\mathrm{S}$. Bloch and H. Esnault [1] in a different way.

Let $Q_{\bullet}\left(F_{\varepsilon}\right)$ be a negatively graded pro-Lie algebra dual to $\mathcal{Q}_{\bullet}\left(F_{\varepsilon}\right)$. Denote by $I_{\bullet}\left(F_{\varepsilon}\right)$ its ideal of elements of degree $\leq-2$, and by $\mathcal{I}_{\bullet}\left(F_{\varepsilon}\right)$ the corresponding Lie coalgebra. Denote by $\mathbb{H}^{*}\left(I_{\bullet}\left(F_{\varepsilon}\right)\right)$ the cohomology of $I_{\bullet}\left(F_{\varepsilon}\right)$ in the category $\mathbb{Q}_{\varepsilon}-\bmod$, i.e. the cohomology of the standard cochain complex $\Lambda^{*} \mathcal{I}_{\bullet}\left(F_{\varepsilon}\right)$ in the category $\mathbb{Q}_{\varepsilon}-$ mod, where we take the exterior powers in $\mathbb{Q}_{\varepsilon}-\bmod$.

Conjecture 1.2. $I_{\bullet}\left(F_{\varepsilon}\right)$ is a free Lie algebra in the category $\mathbb{Q}_{\varepsilon}-\bmod$, with the space of degree $-n$ generators, $n=2,3, \ldots$, given by $\mathcal{B}_{n}(F) \oplus \beta_{n}(F) \cdot \varepsilon$. This means that

$$
\mathbb{H}^{p}\left(I_{\bullet}\left(F_{\varepsilon}\right)\right)=0, \quad p>1 ; \quad \mathbb{H}_{(n)}^{1}\left(I_{\bullet}\left(F_{\varepsilon}\right)\right)=\mathcal{B}_{n}(F) \oplus \beta_{n}(F) \cdot \varepsilon
$$

Here $\mathbb{H}_{(n)}^{1}$ stays for the degree $n$ part of $\mathbb{H}^{1}$. In Section 4.2 we will add one more statement to this conjecture, omitted now for the sake of simplicity. We would like to stress a similarity between this conjecture and the Freeness Conjecture for the mixed elliptic motives, see Conjecture 4.3 in [12]: both conjectures can hardly be formulated without the use of certain esoteric non-rigid tensor structures.

A statement about an object $V \oplus V_{1} \cdot \varepsilon$ in the category $\mathbb{Q}_{\varepsilon}-\bmod$ is actually a pair of statements: one about $V_{0}$, and the other about $V_{1} \cdot \varepsilon$, called the $\mathbb{Q}$ - and $\varepsilon$-parts of the statement.

The $\varepsilon$-part of Conjecture 1.2 is a sophisticated version of Conjecture 1.1. We will show that they are equivalent for $n \leq 3$. Its $\mathbb{Q}$-part is the Freeness Conjecture from [9]-[10] under a scissor congruence hat.

The $\varepsilon$-part of Conjecture 1.2 allows to express explicitly the $F^{*}$-modules $\mathcal{E}_{n}(F)$ via the $\mathbb{Q}$-vector spaces $\mathcal{B}_{n}(F)$ and $F^{*}$-modules $\beta_{n}(F)$.

Examples. One should have

$$
\mathcal{E}_{2}(F)=\beta_{2}(F), \quad \mathcal{E}_{3}(F)=\beta_{3}(F)
$$

The $F^{*}$-module $\mathcal{E}_{4}(F)$ should sit in the exact sequence

$$
0 \longrightarrow \beta_{4}(F) \longrightarrow \mathcal{E}_{4}(F) \longrightarrow \beta_{2}(F) \otimes_{\mathbb{Q}} \mathcal{B}_{2}(F) \longrightarrow 0
$$


1.3. Scissor congruence groups and mixed Tate motives over dual numbers. Below we use the basic facts and terminology from the Tannakian formalism in a mixed Tate category, e.g. the fundamental Hopf and Lie algebras of such a category, see the Appendix in [11] for the background. However the very existence of the Lie algebra $\mathcal{L}_{\varepsilon}$ in the tensor category $\mathbb{Q}_{\varepsilon}$-mod does not follow from this formalism, which works only in the framework of rigid tensor categories, while the category $\mathbb{Q}_{\varepsilon}$-mod is not rigid.

According to [8], Section 1.7, the Hopf algebra $S_{\bullet}(F)$ is isomorphic to the fundamental Hopf algebra of the category $\mathcal{M}_{T}(F)$ of mixed Tate motives over $F$. This means that the category of finite dimensional graded comodules over $S_{\bullet}(F)$ is equivalent to the category of mixed Tate motives over $F$.

Conjecture 1.3. The category of finite dimensional graded comodules over the Lie coalgebra $\mathcal{Q} \cdot\left(F_{\varepsilon}\right)$ is naturally equivalent to a subcategory of the category of mixed Tate motives over the dual numbers $F_{\varepsilon}$.

We show that a simplex in a Euclidean affine space over $F$ provides a comodule over the Lie coalgebra $\mathcal{Q} \bullet\left(F_{\varepsilon}\right)$. It corresponds to a mixed Tate motive over $F_{\varepsilon}$ obtained by perturbation of the zero object over $F$. The subcategory $\mathcal{M}_{T}(F)$ should be given by the comodules with trivial action of $\mathcal{E}_{\bullet}(F)$.

A cycle approach to the mixed Tate motives over $F_{\varepsilon}$ was suggested in [1]. The structure of the paper. The additive polylogarithmic motivic complexes are defined in Section 2. In Section 3 we define Euclidean scissor congruence groups $\mathcal{E}_{n}(F)$. In Section 4 we discuss the category of mixed motives over dual numbers and its relationship with the scissor congruence groups.

\section{Additive polylogarithmic motivic complexes}

2.1. Cathelineau's complexes. Recall the higher Bloch groups $\mathcal{B}_{n}(F)$ defined in [9]-[10]. One has $\mathcal{B}_{1}(F)=F^{*}$.

In [3] J-L. Cathelineau defined the $F$-vector spaces, denoted below by $\bar{\beta}_{n}(F)$. Each of them is generated by the elements $\langle x\rangle_{n}$, where $x \in F$. One has $\bar{\beta}_{1}(F)=$ $F$. The definition goes by induction. For a set $X$ denote by $F[X]$ the $F$-vector space with the basis $\langle x\rangle$, where $x \in X$. Having the $F$-vector spaces $\bar{\beta}_{k}(F)$ for $k<n$ one defines $\bar{\beta}_{n}(F)$ as the quotient of $F\left[F^{*}-\{1\}\right]$ by the kernel of the map of $F$-vector spaces

$$
\delta: F\left[F^{*}-\{1\}\right] \longrightarrow \bar{\beta}_{n-1}(F) \otimes \mathcal{B}_{1}(F) \quad \oplus \quad \bar{\beta}_{1}(F) \otimes \mathcal{B}_{n-1}(F)
$$

given on the generators by

$$
\langle x\rangle \longmapsto\langle x\rangle_{n-1} \otimes\{1-x\}_{1}+\langle 1-x\rangle_{1} \otimes\{x\}_{n-1}
$$

So, by the very definition, there is an injective map of $F$-vector spaces

$$
\delta: \bar{\beta}_{n}(F) \longrightarrow \bar{\beta}_{n-1}(F) \otimes \mathcal{B}_{1}(F) \quad \oplus \quad \bar{\beta}_{1}(F) \otimes \mathcal{B}_{n-1}(F)
$$


Using this let us define the following complex:

$$
\bar{\beta}_{n}(F) \longrightarrow \begin{gathered}
\bar{\beta}_{n-1}(F) \otimes F^{*} \\
F \otimes \mathcal{B}_{n-1}(F)
\end{gathered} \quad \begin{gathered}
\bar{\beta}_{n-2}(F) \otimes \Lambda^{2} F^{*} \\
F \otimes \mathcal{B}_{n-2}(F) \otimes F^{*}
\end{gathered} \longrightarrow \ldots \longrightarrow F \otimes \Lambda^{n-1} F^{*}
$$

It has $n$ terms and placed in degrees $[1, n]$. Its $k$-th term for $k=2, \ldots, n-1$ is

$$
\bar{\beta}_{n-k}(F) \otimes \Lambda^{k} F^{*} \oplus F \otimes \mathcal{B}_{n-k}(F) \otimes \Lambda^{k-1} F^{*}
$$

The differential is defined using $(3)$ and the Leibniz rule. We denote this complex by $\bar{\beta}_{\bullet}(F ; n)$. There is a homomorphism

$$
F \otimes \Lambda^{n-1} F^{*} \longrightarrow \Omega_{F / \mathbb{Q}}^{n-1}, \quad a \otimes b_{1} \wedge \ldots \wedge b_{n-1} \longmapsto a \cdot d \log b_{1} \wedge \ldots \wedge d \log b_{n-1}
$$

It provides a homomorphism

$$
H^{n} \bar{\beta}_{\bullet}(F ; n) \longrightarrow \Omega_{F / \mathbb{Q}}^{n-1}
$$

It follows from the results of [3] that it is an isomorphism. Cathelineau conjectured that $\bar{\beta}_{\bullet}(F ; n)$ is a resolution of $\Omega_{F / \mathbb{Q}}^{n-1}[-n]$, i.e. we have

Conjecture 2.1. For $k<n$ one has

$$
H^{k} \bar{\beta} \cdot(F ; n) \otimes \mathbb{Q}=0
$$

It was proved in [3] that this is the case for $n=3$.

\subsection{Additive polylogarithmic motivic complexes.}

Definition 2.2. The $F^{*}$-module $\beta_{n}(F)$ is defined inductively by

$$
\beta_{n}(F):=\beta_{n-1}(F)\langle 1\rangle \oplus \bar{\beta}_{n}(F)
$$

It follows that we have a decomposition

$$
\beta_{n}(F)=\bar{\beta}_{1}(F)\langle n-1\rangle \oplus \bar{\beta}_{2}(F)\langle n-2\rangle \oplus \ldots \oplus \quad \bar{\beta}_{n}(F)
$$

This is the decomposition into eigenspaces of the $F^{*}$-action.

Example. The first term in (6) is $F\langle n-1\rangle$.

We define a complex $\beta_{\bullet}(F ; n)$ just like $\bar{\beta}_{\bullet}(F ; n)$, but with $\bar{\beta}_{k}(F)$ replaced everywhere by $\beta_{k}(F)$ :

$$
\beta_{n}(F) \longrightarrow \begin{aligned}
& \beta_{n-1}(F) \otimes F^{*} \\
& F \otimes \mathcal{B}_{n-1}(F)
\end{aligned} \longrightarrow \begin{aligned}
& \beta_{n-2}(F) \otimes \Lambda^{2} F^{*} \\
& F \otimes \mathcal{B}_{n-2}(F) \otimes F^{*}
\end{aligned} \longrightarrow \ldots \longrightarrow F \otimes \Lambda^{n-1} F^{*}
$$

Examples. 1. The weight two complex $\beta_{\bullet}(F ; 2)$ is

$$
\beta_{2}(F) \longrightarrow F \otimes F^{*}
$$

2. The weight three complex $\beta_{\bullet}(F ; 3)$ looks as follows:

$$
\beta_{3}(F) \longrightarrow \begin{gathered}
\beta_{2}(F) \otimes F^{*} \\
F \otimes \mathcal{B}_{2}(F)
\end{gathered} \longrightarrow F \otimes \Lambda^{2} F^{*}
$$


Proposition 2.3. Conjecture 2.1 for all weights $\leq n$ is equivalent to the following one: for $i \leq n$ one has

$$
H^{i} \beta_{\bullet}(F ; n)=\Omega_{F / \mathbb{Q}}^{i-1}\langle n-i\rangle
$$

Proof. We have a decomposition into direct sum of complexes

$$
\beta_{\bullet}(F ; n)=\oplus_{k=0}^{n-1} \bar{\beta} \cdot(F ; n-k)\langle k\rangle
$$

The proposition follows.

2.3. Additive versus tangential. Recall that the tangent $T \mathcal{F}$ to a functor $\mathcal{F}$ from a category of rings to an abelian category is defined by

$$
T \mathcal{F}(R):=\operatorname{Ker}\left(\mathcal{F}\left(R[\varepsilon] / \varepsilon^{2}\right) \longrightarrow \mathcal{F}(R)\right)
$$

Problem. Show that Suslin's theorem [15] relating the first cohomology group of the Bloch complex and $K_{3}^{\text {ind }}(F)$ remains valid over the dual numbers.

Using this one could show that the tangent Bloch group $T B_{2}(F)$ is an extension of $\beta_{2}(F)$ by $\Lambda^{2} F$.

So neither $\bar{\beta}_{n}(F)$ nor $\beta_{n}(F)$ are isomorphic to $T \mathcal{B}_{n}(F)$. However assuming Conjecture 2.1 and thanks to Theorem 4.1 , the complex $\beta_{\bullet}(F ; n)$ has the same cohomology as we expect for the tangent motivic complex over $F$. Moreover it should be quasiisomorphic to it. In any case the tangent to the polylogarithmic motivic complex (see [9]-[10]) should be quasiisomorphic to the complex $\beta_{\bullet}(F ; n)$.

Given an $F^{*}$-module $M$ with the $F^{*}$-action written as $f * m$, let $M_{(1)}$ be the maximal submodule of $M$ where the $F^{*}$-action induces the structure of an $F$-vector space, i.e. $(a+b) * m=a * m+b * m$ for any $a, b \in F^{*}$ such that $a+b \in F^{*}$, and any $m \in M$. One sets

$$
M_{(2 k+1)}:=M\langle-k\rangle_{(1)}
$$

It would be interesting to reverse the logic of our definition, and define first $F^{*}$-modules $\beta_{n}(F)$ via the $F^{*}$-modules $T \mathcal{B}_{n}(F)$, and then introduce $F$-vector spaces $\bar{\beta}_{n}(F)$ as follows:

$$
\bar{\beta}_{n}(F):=\beta_{n}(F)_{(1)}
$$

Moreover we should have the decomposition (5), and hence decompositions (6) and (8) into the eigenspaces of the $F^{*}$-action, that is

$$
\beta_{n}(F)_{(2 k+1)}=\bar{\beta}_{n-k}\langle k\rangle, \quad \beta_{n}(F)=\oplus_{k=0}^{n-1} \beta_{n}(F)_{(2 k+1)}
$$

A $K$-theoretic definition of $\beta_{2}(F)$ is given by S. Bloch and H. Esnault in [1]. See also Sections 3.5-3.6 below for $n=2,3$.

\section{The Euclidean scissor congruence groups}

3.1. Euclidean vector spaces. We say that a finite dimensional $F$-vector space has a Euclidean structure if it is equipped with a non-degenerate quadratic form $Q$. A Euclidean affine space is an affine space over a Euclidean vector space.

A Euclidean structure $Q$ on a vector space $V$ provides a Euclidean structure $\operatorname{det}_{Q}$ on $\operatorname{det} V$. A Euclidean volume form in $V$ is a volume form $\operatorname{vol}_{Q}$ such that 
$\operatorname{vol}_{Q}^{2}=\operatorname{det}_{Q}$. Clearly there are two possible choices, $\pm \operatorname{vol}_{Q}$. A choice of one of them is called an orientation of $V$.

Suppose that $V$ is a Euclidean vector space of dimension $2 n$. Then a choice of an orientation of $V$ has the following interpretation. The Euclidean structure provides an operator $*: \Lambda^{\bullet} V \longrightarrow \Lambda^{2 n-\bullet} V$ such that $*^{2}=1$. Namely, if $x \in \Lambda^{k} V$ then for any $y \in \Lambda^{k} V$ one has $\operatorname{vol}_{Q}(* x \wedge y)=<x, y>_{Q}$ where $<>_{Q}$ is the induced Euclidean structure on $\Lambda^{k} V$. The $*$-operator leaves invariant the subspace $\Lambda^{n} V$. Since $*^{2}=1$, there is a decomposition

$$
\Lambda^{n} V^{*}=\Lambda^{n} V_{+}^{*} \oplus \Lambda^{n} V_{-}^{*}
$$

on the \pm 1 eigenspaces of $*$. We call the elements of $\Lambda^{n} V_{+}^{*}\left(\right.$ respectively $\left.\Lambda^{n} V_{-}^{*}\right)$ the selfdual (respectively antiselfdual) $n$-forms. It follows from the very definition that changing the orientation of $V$ we change the $*$-operator by multiplying it by -1 , and thus interchange the selfdual and antiselfdual $n$-forms.

Let $F$ be an algebraically closed field. Then there is an alternative geometric description of this decomposition. The family of $n$-dimensional isotropic subspaces for the quadratic form $Q$ has two connected components. They are homogeneous spaces for the special orthogonal group of $V$, and interchanged by orthogonal transformations with the determinant -1 . The corresponding isotropic subspaces are called the $\alpha$ and $\beta$ planes.

Lemma 3.1. The restriction of any $n$-form from $\Lambda^{n} V_{-}^{*}$ (respectively $\Lambda^{n} V_{+}^{*}$ ) to every isotropic subspace of one (respectively the other) of these families is zero.

Proof. Left as an exercise.

We call the isotropic planes of the first (respectively the second) family the $\alpha$ - (respectively $\beta$-planes). Changing the orientation of $V$ interchanges the $\alpha^{-}$ and $\beta$-planes.

3.2. The Euclidean scissor congruence groups $\mathcal{E}_{n}(F)$. We assume that $F$ is an arbitrary field. Let $A$ be a Euclidean affine space of dimension $2 n-1$. A collection of points $x_{0}, \ldots, x_{2 n-1}$ in $A$ provides a simplex with vertices at these points. We say that such a simplex is Euclidean if the Euclidean structure in $A$ induces a Euclidean structure on each of its faces. The abelian group $\mathcal{E}_{n}(F)$ is generated by the elements $\left(x_{0}, \ldots, x_{2 n-1} ; \operatorname{vol}_{Q}\right)$, where $x_{i} \in A,\left(x_{0}, \ldots, x_{2 n-1}\right)$ is a Euclidean simplex in $A$, and $\operatorname{vol}_{Q}$ is a Euclidean volume form. The relations are the following:

i) (Nondegeneracy). $\left(x_{0}, \ldots, x_{2 n-1} ; \operatorname{vol}_{Q}\right)=0$ if all $x_{i}$ belong to a hyperplane.

ii) (Skew-symmetry). a) $\left(x_{0}, \ldots, x_{2 n-1} ;-\operatorname{vol}_{Q}\right)=-\left(x_{0}, \ldots, x_{2 n-1} ; \operatorname{vol}_{Q}\right)$.

b) For any permutation $\sigma$ one has

$$
\left(x_{0}, \ldots, x_{2 n-1} ; \operatorname{vol}_{Q}\right)=\operatorname{sgn}(\sigma)\left(x_{\sigma(0)}, \ldots, x_{\sigma(2 n-1)} ; \operatorname{vol}_{Q}\right)
$$

iii) (The scissor axiom). For any $2 n+1$ points $x_{0}, \ldots, x_{2 n}$ one has

$$
\sum_{i=0}^{2 n}(-1)^{i}\left(x_{0}, \ldots, \widehat{x}_{i}, \ldots, x_{2 n} ; \operatorname{vol}_{Q}\right)=0
$$


provided that all the simplices involved here are Euclidean.

iv) (Affine invariance). For any affine transformation $g$ of $A$ one has

$$
\left(x_{0}, \ldots, x_{2 n-1} ; \operatorname{vol}_{Q}\right)=\left(g x_{0}, \ldots, g x_{2 n-1} ; g \operatorname{vol}_{Q}\right)
$$

Observe that $g \mathrm{vol}_{Q}$ is a volume form for the quadratic form $g Q$.

Remark. To get the classical Euclidean scissor congruence groups one has to take $F=\mathbb{R}$ and consider only positive definite quadratic forms $Q$ in $\mathbb{R}^{2 n-1}$. Observe that in this case all simplices are Euclidean.

The dilatations provide an action of the group $F^{*}$ on the group $\mathcal{E}_{n}(F)$.

We define the volume of a simplex $S$ spanned by the vectors $v_{1}, \ldots, v_{n}$ in an $n$-dimensional Euclidean space by $\frac{1}{n !}\left\langle v_{1} \wedge \ldots \wedge v_{n}, \operatorname{vol}_{Q}\right\rangle$. The following lemma is straitforward:

Lemma 3.2. The volume of a simplex provides a homomorphism of $F^{*}$-modules

$$
\mathrm{Vol}: \mathcal{E}_{n}(F) \longrightarrow F\langle n-1\rangle
$$

Example. The length provides an isomorphism $\mathcal{E}_{1}(F) \cong F$.

3.3. The scissor congruence Hopf algebra $S_{\bullet}(F)$. We assume that $F$ is an arbitrary field. The definition given below follows s. 3.4 in [8]. Let $V_{2 n}$ is a $2 n$-dimensional $F$-vector space and $Q$ a non-degenerate quadratic form in $V_{2 n}$. Let $M=\left(M_{1}, \ldots, M_{2 n}\right)$ be a collection of codimension one subspaces in $V_{2 n}$. We say that $M$ is in generic position to $Q$ if restriction of the quadratic form $Q$ to any face $M_{I}:=M_{i_{1}} \cap \ldots \cap M_{i_{k}}$ is non-degenerate.

The abelian group $S_{n}(F)$ is generated by the elements $\left(M, Q, \operatorname{vol}_{Q}\right)$, usually denoted simply by $\left(M, \operatorname{vol}_{Q}\right)$, where $\operatorname{vol}_{Q}$ is a volume form for the quadratic form $Q$ and $M$ is a Euclidean simplex with respect to $Q$. The relations are the following:

i) $\left(M, \operatorname{vol}_{Q}\right)=0$ if $\cap M_{i} \neq 0$.

ii) For any $g \in G L\left(V_{2 n}\right)$ one has $\left(M, Q, \operatorname{vol}_{Q}\right)=\left(g M, g Q, g \operatorname{vol}_{Q}\right)$.

iii) $\left(M,-\operatorname{vol}_{Q}\right)=-\left(M, \operatorname{vol}_{Q}\right)$, the skew-symmetry with respect to the permutations of $M_{i}$ 's holds.

iv) For any $2 n+1$ subspaces $M_{0}, \ldots, M_{2 n}$ such that for any $I \subset\{0, \ldots, 2 n\}$ the restriction of $Q$ to $M_{I}$ is non-degenerate, and $M^{(j)}:=\left(M_{0}, \ldots, \widehat{M}_{j}, \ldots, M_{2 n}\right)$, we get

$$
\sum_{j=0}^{2 n}(-1)^{j}\left(M^{(j)}, \operatorname{vol}_{Q}\right)=0
$$

Example. Let $n=1$. Suppose that there is a non zero isotropic vector for $Q$, for instance $F$ is algebraically closed. Then one has $S_{1}(F)=F^{*}$. Indeed, a generator of $S_{1}(F)$ provides an ordered 4-tuple $\left(M_{1}, M_{2}, L_{1}, L_{2}\right)$ of one dimensional subspaces in $V_{2}$. Here $L_{1}$ and $L_{2}$ are the two isotropic subspaces for the form $Q$ ordered so that $L_{1}$ is the $\alpha$-subspace. Then the cross-ratio $r\left(M_{1}, M_{2}, L_{1}, L_{2}\right)$ provides an isomorphism of $S_{1}(F)$ with $F^{*}$.

Remark. In [8] we spelled this definition in a bit different form by considering algebraic simplices in the projective space $P\left(V_{2 n}\right)$ equipped with a non-degenerate 
quadric $Q$, and defining an orientation by choosing one of the families of maximally isotropic subspaces on the quadric $Q$. However these two definitions are equivalent. Indeed, a choice of the Euclidean volume form $\operatorname{vol}_{Q}$ determines the *-operator, and hence, by Lemma 3.1, a choice of one of the families of maximally isotropic subspaces on $Q$.

The commutative, graded Hopf algebra structure on

$$
S_{\bullet}(F):=\oplus_{n \geq 0} S_{n}(F) ; \quad S_{0}(F)=\mathbb{Q}
$$

were defined in theorem 3.9 in [8]. The space of indecomposables $\mathcal{Q} \bullet(F)$ of the Hopf algebra $S_{\bullet}(F)$ (see Section 1.2) has a natural structure of a graded Lie coalgebra with the cobracket $\delta$ inherited from the coproduct.

3.4. The Euclidean Dehn invariant. It is a homomorphism

$$
D^{E}: \mathcal{E}_{n}(F) \longrightarrow \oplus_{k+l=n} \mathcal{E}_{k}(F) \otimes S_{l}(F), \quad k, l>0
$$

Let us define its $\mathcal{E}_{k}(F) \otimes S_{l}(F)$-component $D_{k, l}^{E}$. Choose a partition

$$
\{1, \ldots, 2 n\}=I \cup J ; \quad|I|=2 l
$$

Since $M$ is a Euclidean simplex, $A_{I}:=\cap_{i \in I} M_{i}$ is a Euclidean affine space of dimension $2 k-1$. The hyperplanes $M_{j}, j \in J$ intersect it, providing a collection $\bar{M}_{J}$ of $2 k$ hyperplanes there. Choosing a Euclidean volume form $\alpha_{I}$ in $A_{I}$ we get an element $\left(\bar{M}_{J}, \alpha_{I}\right) \in \mathcal{E}_{k}(F)$.

The quotient $E_{J}:=A / A_{I}$ is a Euclidean vector space of dimension $2 l$. The hyperplanes $M_{i}, i \in I$ project to the collection of hyperplanes $\bar{M}_{I}$ in $E_{J}$. Choose a volume form $\alpha_{J}$ and let $\alpha=\alpha_{I} \otimes \alpha_{J}$. We get an element $\left(\bar{M}_{J}, \alpha_{J}\right) \in S_{l}(F)$. We set

$$
D_{k, l}^{E}(M, \alpha):=\sum_{I}\left(\bar{M}_{J}, \alpha_{I}\right) \otimes\left(\bar{M}_{I}, \alpha_{J}\right)
$$

The statements that $D^{E}$ is a group homomorphism is checked just as Theorem $3.9 \mathrm{a})$ in [8]. It follows easily from the very definitions that

$$
\left(D^{E} \otimes \mathrm{Id}+\mathrm{Id} \otimes D\right) \circ D^{E}=0
$$

Projecting the second component of $D^{E}$ to $\mathcal{Q}_{l}(F)$ we get the reduced Euclidean Dehn invariant

$$
\bar{D}^{E}: \mathcal{E}_{n}(F) \longrightarrow \oplus_{k+l=n} \mathcal{E}_{k}(F) \otimes \mathcal{Q}_{l}(F), \quad k, l>0
$$

It gives rise to the weight $n$ reduced Euclidean Dehn complex $\mathcal{E}_{n}^{*}(F)$ : here "reduced" means that it is obtained from the Euclidean Dehn complex by projecting from $S_{k}(F)$ to $\mathcal{Q}_{k}(F)$ everywhere. The direct sum of the complexes $\mathcal{E}_{n}^{*}(F)$ over $n>0$ is the complex $\mathcal{E}^{*}(F)$ which looks as follows:

$$
\mathcal{E}^{*}(F): \quad \mathcal{E}_{\bullet}(F) \longrightarrow \mathcal{E}_{\bullet}(F) \otimes \mathcal{Q}_{\bullet}(F) \longrightarrow \mathcal{E}_{\bullet}(F) \otimes \Lambda^{2} \mathcal{Q} \bullet(F) \longrightarrow \ldots
$$

Lemma 3.3. a) The Dehn invariant provides $\mathcal{E}_{\bullet}(F)$ with a structure of a comodule over the graded Hopf algebra $S_{\bullet}(F)$.

b) The reduced Dehn invariant provides $\mathcal{E}_{\bullet}(F)$ with a structure of a graded comodule over the Lie coalgebra $\mathcal{Q} \bullet(F)$. 
Proof. a) The proof is similar to the one of Theorem 3.9b) in [8].

b) This is a standard consequence of a). The lemma is proved.

Therefore we get a Lie coalgebra in the category $\mathbb{Q}_{\varepsilon}-\bmod$ :

$$
\mathcal{Q}_{\bullet}\left(F_{\varepsilon}\right):=\mathcal{Q}_{\bullet}(F) \oplus \mathcal{Q}_{\bullet}^{a}\left(F_{\varepsilon}\right) \cdot \varepsilon, \quad \mathcal{Q}_{\bullet}^{a}\left(F_{\varepsilon}\right):=\mathcal{E}_{\bullet}(F)
$$

The standard cochain complex of the Lie coalgebra $\mathcal{Q} \bullet\left(F_{\varepsilon}\right)$ is given by the complex

$$
\mathcal{Q}_{\bullet}\left(F_{\varepsilon}\right) \longrightarrow \Lambda^{2} \mathcal{Q} \bullet\left(F_{\varepsilon}\right) \longrightarrow \ldots \longrightarrow \Lambda^{n} \mathcal{Q} \bullet\left(F_{\varepsilon}\right) \longrightarrow \ldots
$$

in $\mathbb{Q}_{\varepsilon}-\bmod$, where the first map is the cobracket, and the others are defined via the Leibniz rule. The decomposition into $\mathbb{Q}$ - and $\varepsilon$ - components provides a decomposition of this complex into a direct sum of two subcomplexes, called the $\mathbb{Q}$ - and $\varepsilon$-components. It follows from the very definitions that the $\mathbb{Q}$ (respectively $\varepsilon^{-}$) component of $(9)$ is the reduced non-Euclidean (respectively Euclidean) Dehn complex of $F$ :

$$
\Lambda^{*}\left(\mathcal{Q} \bullet\left(F_{\varepsilon}\right)\right)=\mathcal{Q}^{*}(F) \oplus \mathcal{E}^{*}(F) \cdot \varepsilon
$$

Conjecture 3.4. Suppose that $F$ is an algebraically closed field. Then there is canonical isomorphism

$$
\mathbb{H}_{(n)}^{i}\left(\mathcal{Q} \bullet\left(F_{\varepsilon}\right)\right)=\operatorname{gr}_{n}^{\gamma} K_{2 n-i}(F) \otimes \mathbb{Q} \quad \oplus \quad \Omega_{F / \mathbb{Q}}^{n-i}
$$

The $\mathbb{Q}$-part of this isomorphism was conjectured in Section 1.7 in [8]. The new ingredient is the $\varepsilon$-part, which is equivalent to Conjecture 1.1.

We will assume from now on that $F$ is an algebraically closed field.

3.5. The weight two Euclidean Dehn complex. This is the complex

$$
D^{E}: \mathcal{E}_{2}(F) \longrightarrow \mathcal{E}_{1}(F) \otimes S_{1}(F)=F \otimes F^{*}
$$

We expect that the results of Cathelineau, Dupont, and Sah imply that this complex is canonically isomorphic to the additive dilogarithmic complex $\beta_{\bullet}(F ; 2)$, i.e. there should exist canonical isomorphism

$$
l_{2}: \beta_{2}(F) \stackrel{\sim}{\longrightarrow} \mathcal{E}_{2}(F)
$$

which commutes with the coproducts and the volume homomorphisms. In other words it makes the following diagram commute:

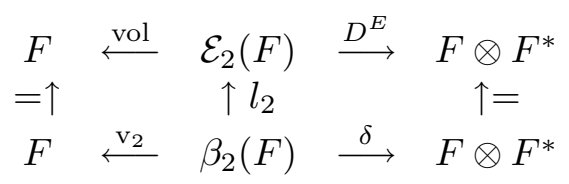


3.6. The weight three reduced Euclidean Dehn complex. This is the complex

$$
\mathcal{E}_{3}(F) \longrightarrow \begin{gathered}
\mathcal{E}_{2}(F) \otimes S_{1}(F) \\
\oplus \\
\mathcal{E}_{1}(F) \otimes \mathcal{Q}_{2}(F)
\end{gathered} \longrightarrow F \otimes \Lambda^{2} F^{*}
$$

We conjecture that the complex (10) is canonically isomorphic to the additive trilogarithmic complex $\beta_{\bullet}(F ; 3)$. This means the following. One should have canonical isomorphism

$$
l_{3}: \beta_{3}(F) \stackrel{\sim}{\longrightarrow} \mathcal{E}_{3}(F)
$$

It should commute with the coproduct and the volume homomorphisms. Finally, combined with the isomorphism $l_{2}$, it should induce an isomorphism of complexes

$$
\begin{aligned}
& \mathcal{E}_{2}(F) \otimes S_{1}(F) \\
& \mathcal{E}_{3}(F) \longrightarrow \mathcal{E}_{1}(F) \otimes \mathcal{Q}_{2}(F) \quad \longrightarrow F \otimes \Lambda^{2} F^{*} \\
& l_{3} \uparrow \quad \uparrow \quad \uparrow= \\
& \beta_{3}(F) \longrightarrow \begin{array}{c}
\beta_{2}(F) \otimes F^{*} \\
\beta_{1}(F) \otimes \mathcal{B}_{2}(F)
\end{array} \longrightarrow F \otimes \Lambda^{2} F^{*}
\end{aligned}
$$

\subsection{The higher reduced Euclidean Dehn complexes.}

Conjecture 3.5. There exist canonical injective homomorphisms of $F^{*}$-modules

$$
l_{n}: \beta_{n}(F) \hookrightarrow \mathcal{E}_{n}(F)
$$

which commutes with the coproduct and the volume homomorphisms.

It follows that the homomorphisms $l_{k}$ for $k \leq n$ provide morphisms of complexes

$$
\beta_{\bullet}(F ; n) \longrightarrow \mathcal{E}^{*}(F ; n)
$$

One can not expect the maps $l_{n}$ to be isomorphisms for $n \geq 4$. Indeed, the restriction of the coproduct to $l_{n}\left(\beta_{4}(F)\right)$ does not have the $\beta_{2}(F) \otimes \mathcal{B}_{2}(F)$ component. The situation for $n>4$ is similar.

\section{The structure of motivic Lie algebras over dual numbers}

4.1. The Tannakian formalism for mixed Tate motives over dual numbers. Recall the Adams filtration $\gamma$ on the $K$-groups. We expect the category $\mathcal{M}_{T}\left(F_{\varepsilon}\right)$ of mixed Tate motives over $F_{\varepsilon}$ to be a mixed Tate $\mathbb{Q}-$ category with the Ext groups given by the formula

$$
\operatorname{Ext}_{\mathcal{M}_{T}\left(F_{\varepsilon}\right)}^{i}(\mathbb{Q}(0), \mathbb{Q}(n))=\operatorname{gr}_{n}^{\gamma} K_{2 n-i}\left(F_{\varepsilon}\right)_{\mathbb{Q}}, \quad A_{\mathbb{Q}}:=A \otimes \mathbb{Q}
$$

The following result was proved in [4], see also Theorem 6.5 in [8]: 
Theorem 4.1. For an arbitrary field $F$ one has

$$
\operatorname{gr}_{\gamma}^{n} K_{2 n-i}\left(F_{\varepsilon}\right) \otimes \mathbb{Q} \quad=\quad \operatorname{gr}_{\gamma}^{n} K_{2 n-i}(F) \otimes \mathbb{Q} \oplus \Omega_{F / \mathbb{Q}}^{n-i}
$$

The projection $F_{\varepsilon} \longrightarrow F$ and inclusion $F \hookrightarrow F_{\varepsilon}$ give rise to the functors

$$
\mathcal{M}_{T}\left(F_{\varepsilon}\right) \longrightarrow \mathcal{M}_{T}(F) ; \quad \mathcal{M}_{T}\left(F_{\varepsilon}\right) \longrightarrow \mathcal{M}_{T}(F)
$$

Therefore the Tannakian formalism implies that there should exist a graded Lie coalgebra $\mathcal{L} \bullet\left(F_{\varepsilon}\right)$ such that the category $\mathcal{M}_{T}\left(F_{\varepsilon}\right)$ of mixed Tate motives over $F_{\varepsilon}$ should be canonically equivalent to the category of finite dimensional graded comodules over $\mathcal{L}_{\bullet}\left(F_{\varepsilon}\right)$. Let us denote by $L_{\bullet}\left(F_{\varepsilon}\right)$ the corresponding Lie algebra. It is a semidirect product of its ideal $L_{\bullet}^{a}\left(F_{\varepsilon}\right)$ and the fundamental Lie algebra $L_{\bullet}(F)$ of the category $\mathcal{M}_{T}(F)$ of mixed Tate motives over $F$ (see the Appendix of [12] for the background). The ideal $L_{\bullet}^{a}(F)$ is not abelian. The group $F^{*}$ acts by the automorphisms of $F_{\varepsilon}: \lambda: a+b \varepsilon \longmapsto a+\lambda b \varepsilon$. So it acts by functoriality on the fundamental Lie algebra $L_{\bullet}\left(F_{\varepsilon}\right)$.

The Ext's (11) can be computed by the weight $n$ part of the standard cochain complex of the Lie coalgebra $\mathcal{L}_{\bullet}\left(F_{\varepsilon}\right)=\mathcal{L}_{\bullet}(F)+\mathcal{L}_{\bullet}^{a}\left(F_{\varepsilon}\right)$, dual to the Lie algebra $L \bullet\left(F_{\varepsilon}\right)$. So using theorem 4.1 we get a conjectural formula

$$
H_{(n)}^{i}\left(\mathcal{L}_{\bullet}\left(F_{\varepsilon}\right)\right)=\operatorname{gr}_{\gamma}^{n} K_{2 n-i}\left(F_{\varepsilon}\right)_{\mathbb{Q}}
$$

Formula (12) contains a lot of information about the fundamental Lie coalgebra $\mathcal{L}_{\bullet}\left(F_{\varepsilon}\right)$. For example, it dictates an isomorphism

$$
\mathcal{L}_{1}\left(F_{\varepsilon}\right)=F_{\varepsilon}^{*} \otimes \mathbb{Q} \cong F_{\mathbb{Q}}^{*} \oplus F
$$

Indeed $\mathcal{L}_{1}\left(F_{\varepsilon}\right)=\operatorname{Ext}_{\mathcal{M}_{T}\left(F_{\varepsilon}\right)}^{1}(\mathbb{Q}(0), \mathbb{Q}(1))=K_{1}\left(F_{\varepsilon}\right)_{\mathbb{Q}}=F_{\varepsilon}^{*} \otimes \mathbb{Q}$. Arguing in a similar way we conclude that one should have isomorphisms

$$
\mathcal{L}_{2}^{a}\left(F_{\varepsilon}\right)=T \mathcal{B}_{2}(F) ; \quad \mathcal{L}_{3}^{a}\left(F_{\varepsilon}\right)=T \mathcal{B}_{3}(F)
$$

One should have the canonical injective maps of $F^{*}$-modules

$$
T \mathcal{B}_{n}(F) \hookrightarrow \mathcal{L}_{n}^{a}\left(F_{\varepsilon}\right)
$$

but they are no longer isomorphisms for $n \geq 4$, just like in the usual case, see [10].

Conjecture 4.2. a) There exists canonical inclusion of Lie coalgebras

$$
\mathcal{Q} \bullet\left(F_{\varepsilon}\right) \hookrightarrow \mathcal{L} \bullet\left(F_{\varepsilon}\right)
$$

b) It induces an isomorphism $\mathbb{H}_{(n)}^{i}\left(\mathcal{Q} \bullet\left(F_{\varepsilon}\right)\right) \stackrel{\sim}{\longrightarrow} H_{(n)}^{i}\left(\mathcal{L}_{\bullet}\left(F_{\varepsilon}\right)\right)$.

c) The Lie subcoalgebra $\mathcal{Q} \bullet\left(F_{\varepsilon}\right)$ is characterized by a) and $\left.b\right)$.

The part a) is nothing else but reformulation of Conjecture 1.3. Theorem 4.1 shows that the $\varepsilon$-part of $\mathrm{b}$ ) is equivalent to Conjecture 1.1 . 


\subsection{The strong version of Freeness Conjecture 1.2. Since}

$$
\frac{Q_{\bullet}\left(F_{\varepsilon}\right)}{I_{\bullet}\left(F_{\varepsilon}\right)}=Q_{-1}\left(F_{\varepsilon}\right), \quad H_{1}\left(I_{\bullet}\left(F_{\varepsilon}\right)\right)=\frac{I_{\bullet}\left(F_{\varepsilon}\right)}{\left[I_{\bullet}\left(F_{\varepsilon}\right), I_{\bullet}\left(F_{\varepsilon}\right)\right]}
$$

we get an action $Q_{-1}\left(F_{\varepsilon}\right) \otimes H_{1}\left(Q_{\bullet}\left(F_{\varepsilon}\right)\right) \longrightarrow H_{1}\left(Q_{\bullet}\left(F_{\varepsilon}\right)\right)$. Dualizing it and using (13) we come to the map

$$
H^{1}\left(I_{\bullet}\left(F_{\varepsilon}\right)\right) \longrightarrow H^{1}\left(I_{\bullet}\left(F_{\varepsilon}\right)\right) \otimes\left(F_{\mathbb{Q}}^{*} \oplus F \cdot \varepsilon\right)
$$

According to Conjecture 1.2 the degree $-n$ part of the $\varepsilon$-component of the map (14) can be identified with the map

$$
\beta_{n}(F) \quad \longrightarrow \quad \mathcal{B}_{n-1}(F) \otimes F_{\mathbb{Q}}^{*} \quad \oplus \quad \beta_{n-1}(F) \otimes F \cdot \varepsilon
$$

We strengthen Conjecture 1.2 by adding to it that this map coincides with (4) after interchanging the factors in the second term.

Proposition 4.3. The strong version of Conjecture 1.2 is equivalent to formula (7) for all $n$, and hence to conjecture 2.1.

Proof. The same argument using the Hochshild-Serre spectral sequence for the ideal $I_{\bullet}\left(F_{\varepsilon}\right)$ as in $[10]$ works. The second statement follows from Proposition 2.3 .

\section{Acknowledgment}

I am grateful to Spencer Bloch for exciting conversations during my visit to U. Chicago at May 2000, which brought me back to the subject. This work was supported by the NSF grant DMS-0099390. I would like to thank the referee for useful comments.

\section{References}

[1] S. Bloch, H. Esnault, An additive version of higher Chow groups. Ann. Sci. Ecole Norm. Sup. (4) 36 (2003), 463-477. math.AG/0112101.

[2] S. Bloch, H. Esnault, The additive dilogarithm. Kazuya Kato"s fiftieth birthday. Doc. Math. Extra Vol. (2003), 131-155. math.AG/0210138.

[3] J-L. Cathelineau, Remarques sur les différentialles des polylogarithmes uniformes. Ann. Inst. Fourier (Grenoble) 46 (1996), 1327-1347.

[4] $\quad \lambda$-structuress in algebraic $K$-theory and cyclic homology. K-theory 4, 6 (1991), 591-606.

[5] _ Sur l'homologie de $S L_{2}$ a coefficients dans l'action adjointe. Math. Scand. 63 (1988), 51-86.

[6] J. Dupont, Algebra of polytopes and homology of flag complexes. Osaka J. of Math. 19 (1982), 599-641.

[7] J. Dupont, H. Sah, Homology of Euclidean groups of motion made discrete and Euclidean scissors congruences. Acta Math. 164 (1990), 1-24.

[8] A.B. Goncharov, Volumes of hyperbolic manifolds and mixed Tate motives. JAMS 12 (1999), 569-618.

[9] _ Geometry of configurations, polylogarithms, and motivic cohomology. Adv. Math. 114 (1995), 197-318. 
[10] _ Polylogarithms and motivic Galois groups. Motives (Seattle, WA, 1991), 43-96, Proc. Sympos. Pure Math., 55, Part 2, Amer. Math. Soc., Providence, RI, 1994.

[11] _ Galois symmetries of fundamental groupoids and noncommutative geometry. ArXiv math.AG/0208144.

[12] _ Mixed elliptic motives. Galois representations in arithmetic algebraic geometry (Durham, 1996), 147-221, London Math. Soc. Lecture Note Ser., 254, Cambridge Univ. Press, Cambridge, 1998. www.math.uiuc.edu/K-theory/ N 228.

[13] T. Goodwillie, Relative algebraic K-theory and cyclic homology. Ann. of Math (2) 124 (1986), 347-402.

[14] H. Sah, Hilbert's third problem: scissors congruences. Research notes in mathematics 33, Pitman Publishing Ltd., San-Francisco-London-Melbourne, 1979

[15] A. Suslin, $K_{3}$ of a field and the Bloch group. Proc. of the Steklov Institute of Mathematics, 183 (1991), 217-240.

[16] J.P. Sydler, Conditions nécessaires et suffisantes pour l'équivalence des polyèdres de l'espace euclidien à trois dimenions. Comment. Math. Helv. 40 (1965), 43-80.

Dept. of Math. Brown University, Providence RI 02912, USA.

E-mail address: sasha@math.brown.edu 